\title{
Manejo de la Fibrilación Atrial
}

\section{Malaria: bibliographic review.}

Pág. 176,185

Recibido: 05-05-2020

Aceptado: 03-07-2020

Dra. Viviana Ruiz Loría1

Dra. Sabrina Acosta Egea2

Dra. Ana Sofía Echeverría Flores 3

1,2,3. Médico General, Trabajador Independiente. San José,Costa Rica.

\section{RESUMEN}

La fibrilación atrial es una taquiarritmia supraventricular con impacto significativo en la salud. Es la arritmia sostenida más común y se caracteriza por provocar palpitaciones, alteraciones a nivel del electrocardiograma y en el pulso del paciente. Comúnmente se asocia a anormalidades cardiovasculares y presenta morbilidad y mortalidad significativas. El manejo del ritmo y la frecuencia tienen el mismo riesgo de evento cerebrovascular y mortalidad, por lo que actualmente se sigue debatiendo sobre cual manejo es prioritario. Es imprescindible evaluar el riesgo cardioembólico, aún tras restaurar un ritmo sinusal, para determinar cuáles pacientes deben ser anticoagulados. Actualmente se prefiere utilizar los anticoagulantes orales de acción directa, sobre la warfarina para los pacientes con fibrilación atrial no valvular. Es importante tratar adecuadamente la fibrilación atrial para reducir los síntomas, prevenir una tromboembolia y una posible cardiomiopatía.

\section{PALABRAS CLAVE}

Fibrilación atrial, arritmia, manejo

\section{ABSTRACT}

Atrial fibrillation is a supraventricular tachyarrhythmia with a significant health impact. It's the most common sustained arrhythmia and it's characterized by causing palpitations, alterations on the electrocardiogram and on its patient's pulse. It is commonly associated with cardiovascular abnormalities and significant morbidity and mortality. The management of rhythm and rate have the same stroke and mortality risk, and it's still currently being debated which management should be prioritized. It is essential that the cardioembolic risk is assessed, even after restoring sinus rhythm, to determine which patients should be anticoagulated. Directacting oral anticoagulants are currently preferred over warfarin for patients with nonvalvular atrial fibrillation. Proper treatment for atrial fibrillation 
is important in order to reduce symptoms, prevent thromboembolisms, and possible cardiomyopathy.

Key words

Atrial fibrillation, arrhythmia, management

\section{INTRODUCCIÓN}

La fibrilación atrial se diagnostica por medio de electrocardiograma con sus características de ausencia de onda $P$, línea de base irregular, presencia de un intervalo $R-R$ variable, frecuencia cardiaca variable y complejo $Q R S$ angosto (1). El manejo actual de la fibrilación atrial está centrado en reducir la morbilidad y la mortalidad que tiene esta patología, así como la mejoría de sintomatología con consecuente disminución de visitas al centro de emergencias y de hospitalizaciones $(2,3,4,5)$. Esta es la causa más común de arritmia cardiaca diagnosticada en los servicios de emergencias en Estados Unidos $(3,4,6)$. Es un factor de riesgo independiente para evento cerebrovascular, insuficiencia cardiaca y para mortalidad $(4,5)$. La clasificación de la misma está compuesta por su duración, su recurrencia y la clínica del paciente. Sus estrategias de manejo se han basado en manejo del ritmo, de la frecuencia y la anticoagulación (7).

La anticoagulación oral con antagonistas de vitamina $\mathrm{K}$ ha sido la base para la prevención de evento cerebrovascular, sin embargo recientemente han salido medicamentos que inhiben los efectos de la trombina y del factor Xa (9). Los objetivos de la presente revisión bibliográfica son explicar el manejo inicial de los pacientes con fibrilación atrial desde el servicio de emergencias, describir las técnicas de manejo de frecuencia, manejo de ritmo y la anticoagulación utilizada.

\section{MÉTODOS:}

La presente revisión bibliográfica se realizó mediante la recopilación y análisis de estudios y artículos basados en evidencia de las bases de datos de Pubmed, Elsevier y Google Scholar. Se seleccionó literatura tanto en inglés como en español. Se utilizaron palabras de búsqueda como "fibrilación atrial," "arritmias," y "manejo." Se utilizaron como criterios de inclusión la validez de las fuentes bibliográficas por hospitales reconocidos a nivel mundial, artículos y estudios realizados por médicos especialistas en el área de interés, y que se hayan realizado en el periodo entre el 2015-2020. Se excluyeron por lo tanto aquellas fuentes con antigüedad anterior al 2015, con excepción a las guías del 2011 y del 2012 de la Sociedad Europea de Cardiología y la American Heart Association.

\section{MANEJO DE LA FRECUENCIA}

Hay una decisión que se debe tomar al presentarse con un caso de fibrilación atrial, y esa es la de ir tras el control del ritmo o de la frecuencia cardiaca (10). Comúnmente, la mayoría de los médicos han preferido el control del ritmo sobre el control de la frecuencia, pero los ensayos clínicos muestran ahora que, comparando ambos, el control del ritmo no mejora en general la mortalidad, la frecuencia de los accidentes cerebrovasculares o la hospitalización, ni la calidad de vida (11).

Hay 3 razones para tratar la fibrilación auricular: reducir los síntomas, prevenir la tromboembolia y prevenir la cardiomiopatía $(1,11)$. Sin importar la estrategia de manejo crónico que se elija, cada paciente con fibrilación atrial detectada de novo debe recibir control de frecuencia ventricular adecuado (10). Una fibrilación atrial 
ventricular rápida sostenida puede llevar a una cardiomiopatía causada por taquicardia $(10,11)$ Los enfoques para tratar a los pacientes con fibrilación auricular de reciente aparición en emergencias varían enormemente (12). La estrategia de "esperar y ver", con cardioversión retrasada si es necesario dentro de las 48 horas siguientes a la aparición de los síntomas, tiene ventajas para los pacientes (12).

La meta de FC para control de frecuencia ha evolucionado con los años $(7,10,11)$. El ensayo prospectivo aleatorio Control de Ritmo vs. Cardio Eléctrico versión II del 2010 estableció que una estrategia de control de ritmo indulgente (objetivo de ritmo cardíaco en reposo, $<110$ latidos/min) es tan efectiva como una estrategia de control de ritmo estricto (objetivo de ritmo cardíaco en reposo, $<80$ latidos $/ \mathrm{min}$ ) $(7,10,11,13)$. Se comparó ambas maneras, y no se vieron diferencias significativas en morbilidad y mortalidad cardiovascular incluyendo la prevalencia de síntomas de fibrilación atrial, hospitalizaciones por falla cardiaca y evento cerebrovascular en pacientes con una fracción de eyección ventricular mayor de $40 \%$ (7). Aún en el escenario de falla cardiaca, disfunción diastólica, cardiomiopatía hipertrófica, hipotensión y congestión pulmonar, no está probado que el control estricto de la frecuencia tenga algún beneficio sobre control permisivo (7). Parámetros de control de frecuencia cardiaca permisivo son más fáciles de obtener en emergencias y mantener en ambulatorio, haciendo esto una opción más simple para el paciente y los profesionales de la salud (7). Para el seguimiento, la frecuencia cardiaca puede ser revisada en citas control o vía un monitor ECG ambulatorio de 24 horas (7).

Para controlar la frecuencia, lo que se hace principalmente es el bloqueo del nodo atrioventricular para limitar la frecuencia de la conducción atrioventricular, y esto se logra con beta bloqueadores, bloqueadores de canales de calcio, y/o digitálicos $(10,11,13)$. Beta bloqueadores con recomendaciones clase 1 son metoprolol, esmolol, y propranolol (7). Posibles efectos adversos son hipotensión, bloqueo cardiaco, bradicardia, broncoespasmo y falla cardiaca (7).

Antagonistas de canales de calcio con recomendaciones clase 1 son diltiazem y verapamilo (7). Ambos tienen perfiles de seguridad similares, incluyendo sus efectos en la presión arterial (7). Diltiazem se usa a dosis de $0.25 \mathrm{mg} / \mathrm{kg}$ intravenoso (IV) en 2 minutes, aunque se ha visto efectividad a dosis menores a $0.2 \mathrm{mg} / \mathrm{kg}$ con menor riesgo de hipotensión (7). Además de la hipotensión, otros efectos adversos posibles son bloqueo cardiaco y falla cardiaca por sus efectos inotrópicos negativos; por lo que no se deben usar en pacientes con falla cardiaca descompensada (7). No tienen efecto adverso de broncoespasmo y son preferidos en pacientes con enfermedad pulmonar obstructiva crónica o asma (7).

La digoxina funciona al aumentar la actividad vagal y el periodo refractario del nodo $\mathrm{AV}$, lo que va a disminuir la frecuencia ventricular (7). El inicio de acción IV de digoxina es mínimo 60 minutos y su efecto pico puede tomar hasta 6 horas; por lo que es de uso limitado en el manejo agudo de fibrilación atrial con respuesta ventricular rápida (7). No es recomendado como agente de primera línea en el manejo fibrilación atrial paroxismal (7). No reduce la taquicardia que se produce con el ejercicio, y es poco probable que controlen la frecuencia en pacientes con insuficiencia cardíaca y alta actividad simpática (11). Es mejor utilizarlo como

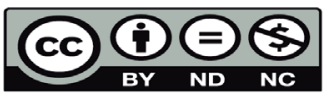


ventricular rápida sostenida puede llevar a una cardiomiopatía causada por taquicardia. $(10,11)$ Los enfoques para tratar a los pacientes con fibrilación auricular de reciente aparición en emergencias varían enormemente (12). La estrategia de "esperar y ver", con cardioversión retrasada si es necesario dentro de las 48 horas siguientes a la aparición de los síntomas, tiene ventajas para los pacientes (12).

La meta de FC para control de frecuencia ha evolucionado con los años. $(7,10,11)$ El ensayo prospectivo aleatorio Control de Ritmo vs. Cardio Eléctrico versión II del 2010 estableció que una estrategia de control de ritmo indulgente (objetivo de ritmo cardíaco en reposo, $<110$ latidos/min) es tan efectiva como una estrategia de control de ritmo estricto (objetivo de ritmo cardíaco en reposo, $<80$ latidos $/ \mathrm{min}$ ) $(7,10,11,13)$. Se comparó ambas maneras, y no se vieron diferencias significativas en morbilidad y mortalidad cardiovascular incluyendo la prevalencia de síntomas de fibrilación atrial, hospitalizaciones por falla cardiaca y evento cerebrovascular en pacientes con una fracción de eyección ventricular mayor de $40 \%$ (7). Aún en el escenario de falla cardiaca, disfunción diastólica, cardiomiopatía hipertrófica, hipotensión y congestión pulmonar, no está probado que el control estricto de la frecuencia tenga algún beneficio sobre control permisivo (7). Parámetros de control de frecuencia cardiaca permisivo son más fáciles de obtener en emergencias y mantener en ambulatorio, haciendo esto una opción más simple para el paciente y los profesionales de la salud (7). Para el seguimiento, la frecuencia cardiaca puede ser revisada en citas control o vía un monitor ECG ambulatorio de 24 horas (7).

Para controlar la frecuencia, lo que se hace principalmente es el bloqueo del nodo atrioventricular para limitar la frecuencia de la conducción atrioventricular, y esto se logra con beta bloqueadores, bloqueadores de canales de calcio, y/o digitálicos $(10,11,13)$. Beta bloqueadores con recomendaciones clase 1 son metoprolol, esmolol, y propranolol (7). Posibles efectos adversos son hipotensión, bloqueo cardiaco, bradicardia, broncoespasmo y falla cardiaca (7).

Antagonistas de canales de calcio con recomendaciones clase 1 son diltiazem y verapamilo (7). Ambos tienen perfiles de seguridad similares, incluyendo sus efectos en la presión arterial (7). Diltiazem se usa a dosis de $0.25 \mathrm{mg} / \mathrm{kg}$ intravenoso (IV) en 2 minutes, aunque se ha visto efectividad a dosis menores a $0.2 \mathrm{mg} / \mathrm{kg}$ con menor riesgo de hipotensión (7). Además de la hipotensión, otros efectos adversos posibles son bloqueo cardiaco y falla cardiaca por sus efectos inotrópicos negativos; por lo que no se deben usar en pacientes con falla cardiaca descompensada (7). No tienen efecto adverso de broncoespasmo y son preferidos en pacientes con enfermedad pulmonar obstructiva crónica o asma (7).

La digoxina funciona al aumentar la actividad vagal y el periodo refractario del nodo $\mathrm{AV}$, lo que va a disminuir la frecuencia ventricular (7). El inicio de acción IV de digoxina es mínimo 60 minutos y su efecto pico puede tomar hasta 6 horas; por lo que es de uso limitado en el manejo agudo de fibrilación atrial con respuesta ventricular rápida (7). No es recomendado como agente de primera línea en el manejo fibrilación atrial paroxismal (7). No reduce la taquicardia que se produce con el ejercicio, y es poco probable que controlen la frecuencia en pacientes con insuficiencia cardíaca y alta actividad simpática (11). Es mejor utilizarlo como adjunto en pacientes con un beta-bloqueador o un antagonista de canales de calcio (7). 
ventricular $(2,7)$. Dofetilide es de la misma clase y se utiliza principalmente en pacientes con fibrilación atrial persistente, sin embargo, por su toxicidad requiere de $72 \mathrm{hrs}$ de monitoreo no invasivo (7). Recientemente se aprobó un nuevo medicamento llamado Vernakalant, el cual es un antiarrítmico atrio selectivo, con una duración de 12 minutos aproximadamente para realizar la cardioversión con efectos adversos como hipotensión y bradicardia (2).

El manejo del ritmo se ha visto que puede funcionar en pacientes con gran sintomatología y en pacientes jóvenes sin cardiopatía estructural (11). Es normal que cuando se presente un paciente joven en un servicio de emergencias con el primer episodio de fibrilación atrial sintomática se prefiera control del ritmo ya que tiene mucha probabilidad de persistir con ritmo sinusal posterior a cardioversión ya que no tiene cardiopatía estructural (11).

Tabla 1. Cardioversión Farmacológica

\begin{tabular}{c|c|c|}
\hline Medicamento & Dosis & Detalle \\
\hline Flecainida & $\begin{array}{c}200 \mathrm{mg} \text { VO o 2mg/kg } \\
\text { IV en 10min }\end{array}$ & $\begin{array}{c}\text { Evitar en } \\
\text { pacientes con } \\
\text { cardiopatía } \\
\text { estructural, } \\
\text { cardiopatía } \\
\text { isquémica o } \\
\text { miocardiopatía }\end{array}$ \\
\hline Propafenona & $\begin{array}{c}600 \mathrm{mg} \text { VO o } 2 \mathrm{mg} / \mathrm{kg} \\
\text { IV en 10 min }\end{array}$ & $\begin{array}{c}\text { Evitar en } \\
\text { pacientes con } \\
\text { cardiopatía } \\
\text { estructural, } \\
\text { cardiopatía } \\
\text { isquémica o } \\
\text { miocardiopatía }\end{array}$ \\
\hline Ibutilide & $\begin{array}{c}1 \mathrm{mg} \text { IV en 10 min } \\
\text { (se puede repetir) }\end{array}$ & $\begin{array}{c}\text { Riesgo de } \\
\text { "torsades de } \\
\text { pointes" }\end{array}$ \\
\hline Amiodarona & $6 \mathrm{mg} / \mathrm{kg}$ IV en $60 \mathrm{~min}$ o \\
$1.2 \mathrm{gr}$ en $24 \mathrm{hrs}$ & $\begin{array}{c}\text { Riesgo de } \\
\text { hipotensión }\end{array}$ \\
\hline
\end{tabular}

\section{ANTICOAGULACIÓN}

La fibrilación atrial aumenta 5 veces el riesgo de presentar un ataque cerebrovascular $y$ este tiene una morbilidad y mortalidad mayor comparado con los eventos cerebrovasculares no relacionados a fibrilación atrial $(7,9,14)$.

Aproximadamente un $80 \%$ de los eventos cerebrovasculares son de tipo isquémicos y de estos $20-30 \%$ se relacionan a fibrilación atrial (14). Los pacientes con esta alteración que tengan una edad entre 80-89 años tienen un riesgo de hasta $8 \%$ de presentar un ataque cerebrovascular (7). Los pacientes con fibrilación atrial que no reciben tratamiento anticoagulante tienen una posibilidad de $0 \%-15,2 \%$ por año o más de presentar un evento tromboembólico (14). Por lo tanto, un tratamiento antitrombótico temprano y adecuado es crítico para estos pacientes (7).Es necesario utilizar una escala para estratificar el riesgo de presentar una embolia en pacientes con fibrilación auricular $(7,14)$.

Actualmente existen varios puntajes que determinan este riesgo y que guían hacia una terapia antitrombótica como; CHADS2, CHA2DS2VASc, ATRIA, Framingham, R2CHADS2, CHA2DS2VASc-R, AFI y SPAF15 $(9,14)$. Sin embargo, el más utilizado es el CHA2DS2VASc (Tabla 2), ya que identifica mejor a los pacientes con muy bajo riesgo tromboembólico que igual se beneficiarían de un tratamiento anticoagulante, que las otras escalas no hacen $(7,9,11,14,15,16)$. Se recomienda iniciar anticoagulación en pacientes con un CHA2DS2VASc $\geq 2$ en hombres $y \geq 3$ en mujeres, mientras que si presenta un CHA2DS2VASc de 0 en hombres y 1 en mujeres no se debe comenzar dicho tratamiento (Tabla 3) (14). El riesgo de sangrado intracraneal se determina

\section{(c) $(1) \Theta \theta$}


mediante las escalas de HEMORR2HAGES, HAS-BLED, ATRIA y ORBIT (14) De estas, la más utilizada es la HAS-BLED, y se utiliza para ayudar a identificar los factores que disponen a eventos hemorrágicos y corregir aquellos que son modificables $(14,16)$ Sin embargo, estas escalas no se deben utilizar para tomar una decisión sobre el inicio o suspensión de la anticoagulación, ya que el riesgo de un evento cerebrovascular supera al riesgo de sangrado $(14,15)$.

\begin{tabular}{|l|l|}
\hline \multicolumn{2}{|l|}{ Tabla 2. Escala $\mathrm{CHA}_{2} \mathrm{DS}_{2}$ VASc } \\
\hline Características & Puntaje \\
\hline Insuficiencia cardiaca congestiva & 1 \\
\hline Hipertensión arterial & 1 \\
\hline Edad $\geq 75$ años & 2 \\
\hline Diabetes mellitus & 1 \\
\hline ICTUS o Accidente isquémico & 2 \\
transitorio & 1 \\
\hline Enfermedad vascular & 1 \\
\hline Edad 65-74 & 1 \\
\hline Sexo femenino & \\
\hline ente: Zimetbaum P. In the Clinic: atrial fibrillation (vol 153, ITC61-15, 2010) \\
\hline nn Intem Med. 2017 Jun 20;166(12):920. Dol: 10.7326/AIITC201703070 \\
\hline
\end{tabular}

Tabla 3. Guías para Profilaxis Tromboembólica Acorde a la Escala $\mathrm{CHA}_{2} \mathrm{DS}_{2}$ VASc

\begin{tabular}{|c|c|}
\hline Escala $\mathrm{CHA}_{2} \mathrm{DS}_{2}$ VASc & Recomendaciones \\
\hline 0 & No se requiere terapia \\
\hline 1 & $\begin{array}{l}\text { No se requlere terapia pero tratamiento } \\
\text { con aspirina o un anticoagulante } \\
\text { (warfarina, dibagatrán, rivaroxabán, } \\
\text { apixabán o edoxabán) es también } \\
\text { razonable }\end{array}$ \\
\hline 20 más & $\begin{array}{l}\text { Anticoagulación con warfarina, } \\
\text { dibagatrán, rivaroxabán, apixabán o } \\
\text { edoxabán }\end{array}$ \\
\hline
\end{tabular}

Los pacientes que presentan una fibrilación atrial no valvular por mayor a 48 horas tienen un riesgo aumentado de un tromboembolismo y un evento cerebrovascular, mientras que aquellos que lo presentan por menos de 48 horas tienen una posibilidad de menos de un $1 \%(7,13)$. Si los pacientes se encuentran hemodinámicamente estables y la fibrilación auricular lleva menos de 48 horas se puede realizar una cardioversión sin necesidad de anticoagulación, pero si llevan más de 48 horas se debe anticoagular al paciente por 3 semanas antes del procedimiento y después por 4 semanas tras la cardioversión eléctrica o farmacológica $(7,16)$. Se recomienda realizar una ecocardiografía transesofágica para evidenciar posibles trombos en el atrio izquierdo, y si este es el caso, se debe mantener la anticoagulación por 3 semanas para luego repetir la ecocardiografía $(7,13)$. Aunque se devuelva al paciente a un ritmo sinusal, esto no garantiza que este ritmo se mantenga y por lo tanto hay que entender que el paciente permanece en riesgo para eventos tromboembólicos y se debe tratar adecuadamente (7).

La warfarina es el único medicamento aprobado para una fibrilación atrial valvular, y por muchos años también lo fue para las no valvulares $(9,14)$ Sin embargo, actualmente en pacientes sin contraindicaciones, la primera línea de tratamiento para fibrilación atrial no valvular son los anticoagulantes orales de acción directa por sus mayores beneficios (14). La warfarina tiene una ventana terapéutica estrecha, numerosas interacciones farmacológicas y alimentarias, no es costo efectiva a largo plazo y necesita una monitorización estricta por su alto riesgo de sangrado $(9,17)$..También, una gran dificultad de este medicamento es mantener el índice internacional normalizado entre 2 y 3 , el cual solo se logra en $2 / 3$ de los ensayos de prueba 
que se han realizado $(7,14)$. Por lo tanto, es importante mantener un control estricto al inicio de la terapia (7).

Los anticoagulantes orales de acción directa que se utilizan actualmente son; dabigatrán, rivaroxabán, apixabán y edoxabán (Tabla 4) (14). Todos estos previenen eventos tromboembólicos en pacientes con fibrilación atrial pero se debe escoger según el tipo de paciente y el escenario (14). Pacientes con alto riesgo de sangrado gastrointestinal se recomienda comenzar con apixaban o dabigatrán (14). Si tienen alto riesgo de sangrado mayor se utiliza apixaban, dabigatrán o edoxabán (14). Si ocurre un evento cerebrovascular a pesar de estar en tratamiento con warfarina se sugiere utilizar dabigatrán (14). Pacientes con quejas gastrointestinales se debe iniciar con apixabán, rivaroxabán o edoxabán (14). Dabigatrán fue el único inhibidos directo de trombina que demostró una disminución en la probabilidad de un evento cerebrovascular o un embolismo sistémico al utilizar $110 \mathrm{mg}$ dos veces al día comparado con los pacientes utilizando warfarina (7). Los anticoagulantes orales de acción directa son no reversibles, por lo tanto se debe evaluar el riesgo/beneficio antes de comenzar el tratamiento $(7,17)$.
Los antiagregantes comola aspirina o clopidogrel, no se recomiendan para reemplazar a los anticoagulantes ya que son menos eficientes para prevenir los eventos cerebrovasculares y tienen una tasa de sangrado similar a los anticoagulantes (14) Estos medicamentos se deben utilizar únicamente en pacientes que presentan alergia hacia la warfarina o los anticoagulantes orales de acción directa, no toleran los anticoagulantes, se rehúsan a utilizarlos, no tienen historia previa de un evento cerebrovascular o un ataque isquémico, son menores de 65 años, no tienen hipertensión, diabetes o fallas cardíacas $(7,11)$.

Según las guías del 2012 de la Sociedad Europea de Cardiología y las del American Heart Association del 2011, se recomienda utilizar clopidogrel junto a la aspirina en los pacientes que el médico contraindica el uso de anticoagulantes o el paciente rehúsa utilizar estos medicamentos, ya que se ha observado que el uso de estos dos tratamientos juntos reduce más la posibilidad de un evento cerebrovascular $(7,11)$. Sin embargo, de igual forma esta combinación no es igual de efectiva que la warfarina $(7,11)$.

\begin{tabular}{|c|c|c|c|c|}
\hline & Dabigatrán & Rivaroxabán & Apixabán & Edoxabán \\
\hline Mecanismo & $\begin{array}{l}\text { Inhibidor directo } \\
\text { de trombina }\end{array}$ & $\begin{array}{l}\text { Inhibidor Factor } \\
\mathrm{Xa}_{\mathrm{a}}\end{array}$ & $\begin{array}{l}\text { Inhibidor Factor } \\
\mathrm{Xa}\end{array}$ & $\begin{array}{l}\text { Inhibidor Factor } \\
\mathrm{Xa}\end{array}$ \\
\hline Dosis & $150 \mathrm{mg}$ BID & $\begin{array}{l}20 \mathrm{mg} c / \mathrm{d} \text { con } \\
\text { comidas }\end{array}$ & $5 \mathrm{mg}$ BID & $\begin{array}{l}60 \mathrm{mg} \text { cid si } \\
\text { paciente con } \mathrm{ClCr} \\
>50 \text { y }<95 \\
\mathrm{~mL} / \mathrm{min}\end{array}$ \\
\hline Contraindicaciones & $\begin{array}{l}\mathrm{ClCr}<15 \mathrm{~mL} / \mathrm{min} \\
\text { o válvula } \\
\text { cardiaca artificial }\end{array}$ & $\begin{array}{l}\mathrm{CICr}<15 \mathrm{~mL} / \mathrm{min} \\
\text { o válvula } \\
\text { cardiaca artificial }\end{array}$ & $\begin{array}{l}\mathrm{ClC} r<15 \mathrm{~mL} / \mathrm{min} \\
\text { o válvula } \\
\text { cardiaca artificial }\end{array}$ & $\begin{array}{l}\mathrm{CICr}>95 \mathrm{~mL} / \mathrm{min} \\
\text { o válvula } \\
\text { cardiaca artificial }\end{array}$ \\
\hline \multicolumn{5}{|c|}{ 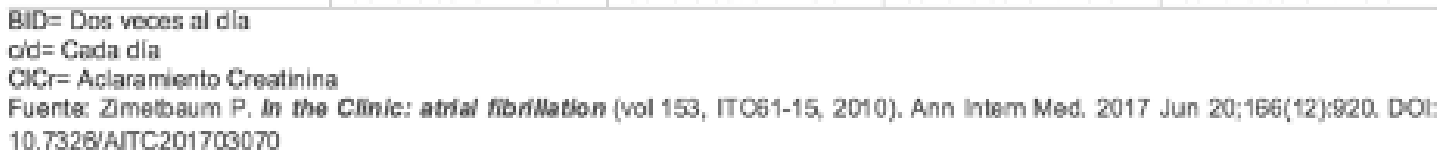 } \\
\hline
\end{tabular}




\section{CONCLUSIÓN}

La fibrilación atrial es una patología sumamente común en los servicios deemergencias. El manejo de la misma es importante para determinar cuál opción tendrá menor morbi-mortalidad para el paciente, utilizando determinantes como recurrencia, edad, comorbilidades, etc. Actualmente se sigue debatiendo si se debe priorizar el manejo del ritmo versos el manejo de la frecuencia, pero al final depende de la disponibilidad de medicamentos en el centro hospitalario y la preparación médica del personal para la realización de los procedimientos. Cabe destacar que la anticoagulación es necesaria en aquellos pacientes con una fibrilación atrial de mayor a 48 horas por su alta posibilidad de presentar un evento cerebrovascular. Los anticoagulantes orales de acción directa son actualmente los medicamentos de primera línea por sus mayores beneficios y control menos estricto. Sin embargo, la warfarina sigue siendo el único medicamento aprobado para las fibrilaciones atriales valvulares. El abordaje completo y oportuno con estas medidas terapéuticas, es imprescindible para obtener un pronóstico favorable y prevenir daños irreversibles a futuro.

\section{CONFLICTOS DE INTERÉS}

Los autores declaran que no existen conflictos de intereses ni beneficios económicos al realizar este artículo.

\section{BIBLIOGRAFÍA}

1. Forero-Gómez JE, Moreno JM, Agudelo CA, Rodríguez-Arias EA, Sánchez-Moscoso PA. Fibrilación auricular: enfoque para el médico no cardiólogo. latreia. 2017 Oct Dic;30(4):404422. DOI 10.17533/udea.iatreia.v30n4a05

2. Andrade JG, Verma A, Mitchell LB, Parkash R, Leblanc K, Atzema C, Healey JS, Bell A, Cairns J, Connolly S, Cox J. 2018 focused update of the Canadian Cardiovascular Society guidelines for the management of atrial fibrillation. Can J Cardiol. 2018 Nov 1;34(11):1371-92. DOI 10.1016/j.cjca.2018.08.026

3. Heidenreich PA, Solis P, Estes NM, Fonarow GC, Jurgens CY, Marine JE, McManus DD, McNamara RL. 2016 ACC/AHA clinical performance and quality measures for adults with atrial fibrillation or atrial flutter: a report of the American College of Cardiology/American Heart Association Task Force on Performance Measures.J. Am. 2016 Aug 2;68(5):525-68.

DOI: 10.1016/j.jacc.2016.03.521

4. Ferreira C, Providência R, Ferreira MJ, Gonçalves LM. Atrial fibrillation and non-cardiovascular diseases: a systematic review. Arq Bras Cardiol. 2015 Nov;105(5):519-26.

DOI: 10.5935/abc.20150142 
5. Bhatia, S., Sugrue, A. and Asirvatham, S., 2018. Atrial Fibrillation: Beyond Rate Control. Mayo Clin Proc. March 2018;93(3):373-380

DOI: 10.1016/j.mayocp.2017.10.024

6. Burashnikov A, Antzelevitch C. Pathophysiology of Atrial Fibrillation. Springer International Publishing. 2017. (2):15-25. DOI 10.1007/978-3-319-58000-5_2

7. Goralnick E, Bontempo L. Atrial Fibrillation. Emerg Med Clin N Am. 2015August;33(3):597-612. DOI: 10.1016/j.emc.2015.04.008

8. Calkins H, Hindricks G, Cappato R, Kim YH, Saad EB, Aguinaga L, Akar JG, Badhwar V, Brugada J, Camm J, Chen PS. 2017 HRS/EHRA/ECAS/APHRS/SOLAECE expert consensus statement on catheter and surgical ablation of atrial fibrillation: executive summary. Journal of Arrhythmia. 2017 Oct;33(5):369-409. DOI: 10.1016/j.joa.2017.08.001

9. Proietti M, Lip GY. Atrial fibrillation and stroke: making sense of recent observations on anticoagulation. Cardiol clin. 2016 May 1;34(2):317-28.

DOI: $10.1016 /$ j.ccl.2015.12.006

10. Morin DP, Bernard ML, Madias C, Rogers PA, Thihalolipavan S, Estes III NM. The state of the art: atrial fibrillation epidemiology, prevention, and treatment. Mayo Clin Proc. 2016 Dec 1 (Vol. 91, No. 12, pp. 1778-1810). Elsevier.

DOI: 10.1016/j.mayocp.2016.08.022

11. Zimetbaum P. In the Clinic: atrial fibrillation (vol 153, ITC61-15, 2010). Ann Intern Med. 2017 Jun 20;166(12):920-.

DOI: $10.7326 /$ AITC201703070

12. Pluymaekers NA, Dudink EA, Luermans JG, Meeder JG, Lenderink T, Widdershoven J, Bucx JJ, Rienstra M, Kamp O, Van Opstal JM, Alings M. Early or delayed cardioversion in recent-onset atrial fibrillation. N Engl J Med. 2019 Apr 18;380(16):1499-508. DOI: 10.1056/ NEJMoa1900353

13. Andrade JG, Macle L, Nattel S, Verma A, Cairns J. Contemporary atrial fibrillation management: a comparison of the current AHA/ACC/HRS, CCS, and ESC guidelines. Can J Cardiol. 2017 Aug 1;33(8):965-76. DOI: 10.1016/j.cjca.2017.06.002

12. Gómez-Rosero JA, Sierra-Vargas EC. Verdades y controversias de la anticoagulación en fibrilación auricular no valvular. Rev Colomb de Cardiol. 2019 Oct 11.

DOI: 10.1016/j.rccar.2019.07.006

\section{() $(1) \Theta \theta$}


15. Freedman B, Potpara TS, Lip GY. Stroke prevention in atrial fibrillation. Lancet. 2016 Aug 20;388(10046):806-17.DOI: 10.1016/S0140-6736(16)31257-0

16. Poveda Rodriguez CA, García García LA, Trejos Hernández J, Villegas Sequiera LR, Vásquez M, Alonso E, González Castro D. Tratamiento agudo de la fibrilación auricular. Rev Med Costa Rica Centroam. 2016 Aug 15;73(620):677-81.

17. Chen ST, Patel MR. Comparison of anticoagulant therapy for atrial fibrillation-novel oral anticoagulants versus vitamin K antagonists. Prog Cardiovasc Dis. 2018 Jan 1;60(4-5):514-23.

DOI: 10.1016/j.pcad.2018.01.005 